\title{
Actividad sexual en personas mayores
}

\section{Sexual activity in older people}

\author{
Isabel Piñeiro, Susana Rodríguez, Sonia Rodríguez, Bibiana Regueiro, Iris Estévez, Marcia Ullauri \\ Universidade da Coruña, España
}

\begin{abstract}
Resumen
Se estudia la frecuencia de las prácticas sexuales y la actitud hacia las relaciones sexuales así como conocer si el lugar donde vive condiciona su actividad sexual de una muestra de 76 personas entre 65 y 90 años. Los resultados revelan que un $33 \%$ de hombres y un $25 \%$ de mujeres afirman haber tenido actividad sexual en el último año. La mayoría de mujeres y hombres afirman que ser activo sexualmente es física y psicológicamente beneficioso. La frecuencia de las prácticas sexuales de los hombres que viven en residencias es significativamente menor que los que viven en su domicilio familiar.
\end{abstract}

Palabras clave: envejecimiento, sexualidad, comportamiento sexual, residencias.

\section{Abstract}

We study the frequency of sexual practices and the attitude towards sexual relations of a sample of 76 elderly people aged between 65 and 90 years. In addition, it seeks to know if the place where the elderly person lives (family home or residence) conditions their sexual activity. The results reveal that $33 \%$ of men reported having had sexual activity in the last year, and in the case of women, $25 \%$. Most women (67.5\%) and men (63.9\%) say that being sexually active is physically and psychologically beneficial. The frequency of sexual practices of men living in residences is significantly lower than those living in their family home.

Keywords: aging, sexuality, sexual behavior, residences.

En los últimos años, se ha producido un aumento del número de personas mayores como resultado del desarrollo de la humanidad, de sus avances educativos, científicos, tecnológicos y socio-políticos, hecho que exige atención a las necesidades de un mundo envejecido (Fernández-Ballestetos, 2006).

En numerosas ocasiones, la vejez ha sido considerada como un proceso de pérdida asociándola con la enfermedad, la discapacidad o la dependencia y con un proceso irreversible que se desencadena en un deterioro físico y psicológico irrecuperable. Como consecuencia, aparecen estereotipos que consideran que dicha merma de capacidades lleva, entre otras cosas, a un deterioro en la capacidad sexual y a la falta de deseo sexual. Entre los numerosos estereotipos vinculados a la vejez se encuentra la consideración de que el sexo ha dejado de tener interés para las personas mayores (Freire y Ferradás, 2016).
La sexualidad constituye un elemento importante en el desarrollo evolutivo de cualquier persona, en sus relaciones interpersonales $\mathrm{y}$ en su salud general. Representa una dimensión fundamental para el bienestar y la calidad de vida de las personas a lo largo de toda su vida (Pérez, 2006).

En realidad, en la población mayor ocurren una serie de cambios que pueden condicionar de manera importante la sexualidad en la vejez pero no, necesariamente, anularla. Para aportar más información sobre esta temática, presentamos la siguiente investigación cuyo objetivo general ha sido conocer el comportamiento sexual de las personas mayores, tanto de las que viven en una residencia como las que viven en su domicilio familiar. Específicamente, nos interesa explorar sus actitudes sexuales; conocer su práctica sexual; conocer el nivel de satisfacción con su vida sexual; y conocer si el lugar de residencia condiciona la actividad sexual de las personas mayores.

\section{Método}

\section{Participantes}

La muestra está formada por 76 personas mayores (hombres y mujeres) de la provincia de Lugo (España), de las cuales 40 viven en una residencia y 36 viven en su domicilio familiar. La muestra masculina estaba compuesta por 36 hombres jubilados, de nacionalidad española y residentes en la provincia de Lugo. Su media de edad era de 78.61 (DT=7.05), abarcando desde los 66 hasta los 89 años. En cuanto a la muestra femenina, está formada por 40 mujeres jubiladas de nacionalidad española y residentes en la provincia de Lugo. La media de edad es de 77.9 (DT=7.575), abarcando desde los 65 hasta los 90 años, predominando las mujeres de 83 años.

\section{Instrumentos}

El instrumento seleccionado para realizar esta investigación ha sido una traducción del SRA-Q (Lee, Nazroo, O'Connor, Blake, y Pendleton, 2016), llevada a cabo por el Grupo de Investigación en Psicología Educativa (GIPED) de la Universidad de A Coruña. En esta traducción se han adaptado los ítems originales, dirigidos a la población británica, a la población española. El instrumento original está constituido a partir de un otros instrumentos validados (Mitchell, 
Ploubidis, Datta y Wellings, 2012; O’Connor et al., 2008; Waite, Laumann, Das y Schumm, 2009).

Se pretende asegurar la especificidad de género, existiendo un cuestionario dirigido a hombres y otro dirigido a mujeres. En total, la escala para hombres está compuesta por 50 ítems y la escala para mujeres por un total de 44 ítems. En ambos se incluye una parte inicial que permite conocer la actitud sobre diferentes aspectos vinculados a la sexualidad. El cuestionario incluye varios ítems relativos al impulso o el deseo sexual propio, así como con otros acerca de la frecuencia de su práctica sexual. En la escala masculina se pregunta por la capacidad de erección, así como a las mujeres sobre su capacidad de excitación sexual. El instrumento dispone también en ambas versiones de ítems relativos al orgasmo y de su vida sexual general durante los últimos tres meses, también de las experiencias sexuales a lo largo de su vida.

Respondiendo a los objetivos planteados, se han analizado la actitud hacia la sexualidad, la práctica sexual en el último año, las diferencias en la actividad sexual en función del lugar de residencia y el nivel de satisfacción con su vida sexual general en los últimos tres meses.

La actitud hacia la sexualidad ha sido medida con los 13 primero ítems de la escala, iguales en ambas versiones del cuestionario. Se presentan en una escala Likert de cinco puntos desde 1 (En total desacuerdo) a 5 (Totalmente de acuerdo).

La práctica sexual en el último año se ha evaluado con el ítem En el último año, ¿ha tenido usted alguna práctica sexual (sexo coital, masturbación, roces o caricias)? del cuestionario que nos permitirá acceder a aquellas preguntas que hablen sobre la implicación en relaciones sexuales, así como en otras prácticas, además de conocer si ha cambiado la frecuencia general de esta actividad sexual. Se presenta con una escala de respuesta dicotómica sílno que hemos codificado como Práctica sexual Sí - en caso de haber mantenido prácticas sexuales - o Práctica sexual No, en el caso contrario.

Para conocer si existen diferencias en la actividad sexual en función del lugar de residencia se ha utilizado, nuevamente, el ítem En el último año, ¿ha tenido usted alguna práctica sexual (sexo coital, masturbación, roces o caricias)? Con la misma codificación y partiendo de los datos proporcionados por la variable demográfica que pregunta por el lugar de la vivienda.

Para conocer el nivel de satisfacción con su vida sexual general en los últimos tres meses se empleó el ítem Durante los últimos tres meses, ¿cómo de satisfecho/a ha estado usted con su vida sexual general? con una escala de respuesta de 5 puntos donde 1 (muy satisfecho/a) a 5 (Muy insatisfecho/a).

Además del SRA-Q, se han incluido ítems que permite conocer diferentes datos sociodemográficos de la muestra (preferencias sexuales, nivel económico y educativo, situación laboral, etc.), así como sobre la percepción de su salud general y sexual que resultan de interés para la presente investigación.

\section{Técnicas de análisis de datos}

Con objeto de dar respuesta a los objetivos de esta investigación se ha llevado a cabo un análisis descriptivo de la muestra para presentar la información sobre las actitudes sexuales, la actividad sexual y la satisfacción sexual.

Para comparar la actividad sexual de la muestra que convive en residencia y la que vive en su domicilio familiar estudiamos la homogeneidad de ambas muestras a partir de tablas de contingencia a través del contraste Chi-cuadrado, ya que las dos variables son categóricas dicotómicas. Para la realización de todos estos análisis se ha utilizado el paquete estadístico SPSS (Versión 20).

\section{Resultados}

A continuación, se exponen los resultados diferenciando entre aquellos relativos a las actitudes hacia la sexualidad, a la práctica sexual, al lugar de residencia como posible condicionante de la práctica sexual y al nivel de satisfacción con la vida sexual general.

\section{Actitud hacia la sexualidad entre las personas mayores.}

Para responder al primer objetivo específico y conocer las actitudes sexuales de las personas mayores, analizamos los resultados obtenidos en los ítems que componen el primer bloque de la escala, donde se presentan una serie de afirmaciones sobre sexualidad, a través de un análisis descriptivo de las respuestas aportadas por las dos muestras con las que se ha trabajado.

En cuanto al primer ítem Tener relaciones sexuales antes del matrimonio es malo, la mayoría de hombres $(83,3 \%)$ está en "desacuerdo" o "total desacuerdo". En el caso de las mujeres, el $45 \%$ está "totalmente de acuerdo" con esta afirmación y un $45 \%$ está "en desacuerdo" o "total desacuerdo".

Existe un alto porcentaje de hombres $(61,1 \%)$ que están "de acuerdo" o "totalmente de acuerdo" con la afirmación Que una persona casada tenga relaciones sexuales con otra que no es su esposa es malo, mientras que un 30,5\% están en "desacuerdo" o "totalmente en desacuerdo". Al igual que los hombres, la mayoría de las mujeres $(72,5 \%)$ están "totalmente de acuerdo" con que es malo tener relaciones sexuales con otra persona que no sea su pareja.

Un alto porcentaje de hombres $(72,2 \%)$ considera que tener sexo de una sola noche no es malo, mientras que el resto mantiene que este comportamiento es negativo. En el caso de las mujeres, están "en desacuerdo" o en "total desacuerdo" con esta afirmación el 37,5\%, mientras que un $42,5 \%$ está "totalmente en desacuerdo" con la acepción de Tener sexo de una sola noche es malo. A la hora de opinar sobre si Las relaciones sexuales sin amor son correctas, un $33,3 \%$ de los hombres y un $52,5 \%$ de las mujeres afirman estar "en desacuerdo" con esta conducta.

Cuando se relacionan las relaciones sexuales satisfactorias con el mantenimiento de una relación a 
largo plazo, la mayoría de las personas, tanto hombres $(97,3 \%)$ como mujeres (95\%) opinan estar de acuerdo y que estas relaciones sexuales son esenciales para la pareja.

Se analiza la opinión que tiene nuestra muestra sobre las personas homosexuales a partir de las respuestas aportadas al ítem Que dos adultos del mismo sexo tengan relaciones sexuales es malo. En ambas muestras el número de personas que está de acuerdo con esta afirmación es prácticamente el mismo en el caso de los hombres $(88,9 \%)$ y en el de las mujeres $(87,5 \%)$.

Se estudia, también, la opinión que los participantes tienen sobre la presión social que experimenta la población para mantener relaciones sexuales, así como su opinión sobre la presencia de la sexualidad en la sociedad actual y en los medios de comunicación. Con la afirmación Hoy en día la gente está bajo mucha presión para tener sexo, el $72 \%$ de las mujeres está "de acuerdo" y, en el caso de la muestra masculina, un $61 \%$ está "totalmente de acuerdo".

Sobre la afirmación de que hay demasiado sexo en los medios de comunicación hoy en día, el 38,9\% de hombres está "de acuerdo" o "totalmente de acuerdo" con este enunciado, el 30,6\% está "en desacuerdo" o "en total desacuerdo", y el $30,6 \%$ se posiciona en la opción de respuesta intermedia "más de acuerdo que en desacuerdo". En el caso de la muestra femenina, es el $62,5 \%$ las que afirman estar "de acuerdo" o "totalmente de acuerdo" con una presencia excesiva del sexo en los medios de comunicación, el resto de la muestra femenina están "en desacuerdo" o "en total desacuerdo" $(17,5 \%)$ y en "más de acuerdo que en desacuerdo" (20\%) con esa afirmación.

Cuando se afirma que hoy la gente joven empieza a tener relaciones sexuales demasiado pronto, tanto la mayoría de la muestra masculina $(86,1 \%)$ como, en el caso de las mujeres, la totalidad de la muestra, están "de acuerdo" o "totalmente de acuerdo" con dicha afirmación.

Con la afirmación Los hombres tienen de forma natural un deseo sexual más alto que las mujeres, la mayoría de la muestra masculina está "de acuerdo" o "totalmente de acuerdo" con ella $(63,9 \%)$ y, en la muestra femenina, es, también, la mayoría la que está "de acuerdo" o "totalmente de acuerdo" (75\%) con el enunciado.

En el ítem que afirma que la capacidad de tener relaciones sexuales disminuye a medida que una persona envejece, aparece casi una unanimidad en las respuestas. Cerca del $93 \%$ de las mujeres indican estar "de acuerdo" o "totalmente de acuerdo" con esta afirmación; en el caso de los hombres son cerca del $90 \%$ los que están "de acuerdo" o "totalmente de acuerdo".

Sin embargo, cuando se alega que los cambios sexuales que ocurren con la edad no son importantes en la gente mayor, aparece un alto porcentaje en ambas muestras que indica estar "en desacuerdo" con ello. De esta manera, el $80,6 \%$ de los hombres y el $80 \%$ de las mujeres están "en desacuerdo" o en "total desacuerdo" con esta afirmación.

En el último ítem de esta primera parte, se afirma que ser activo sexualmente es física y psicológicamente beneficioso para la gente mayor. Tanto la mayoría de los hombres $(91,7 \%)$ y como de las mujeres $(90 \%)$ están "de acuerdo" o "totalmente de acuerdo" con esta consideración.

\section{Frecuencia de la práctica sexual entre los mayores.}

Para conocer la práctica sexual de la muestra realizamos un análisis descriptivo de las respuestas dadas a los ítems relacionados con la práctica sexual. Describimos las respuestas de los 12 hombres y 10 mujeres que han tenido alguna práctica sexual en el último año.

Durante el mes pasado, ¿cuántas veces ha tenido usted o intentado mantener relaciones sexuales (vaginal, anal o sexo oral)?

De entre la muestra masculina, la media de respuesta a esta pregunta es de 2.75 (DT=1.712), siendo la escala de respuesta 1 "Ninguna" y la 5 "Más de una vez al día". El 13,9\% de estas 12 personas afirma no haber tenido ninguna práctica en el último mes, un $8,3 \%$ las han mantenido o intentado mantenerlas 2 o 3 veces en el mes pasado, porcentaje similar a aquellos que han llevado a cabo práctica sexual 2 o 3 veces por semana. En la muestra femenina, en el último mes, el 12,5\% afirman no haber tenido o intentado mantener relaciones sexuales, el $2,5 \%$ indica haber mantenido relaciones sexuales una vez al mes, el 7,5\% dos o tres veces al mes y otro $7,5 \%$ afirma haberlas mantenido o intentado una vez a la semana.

A parte de las veces que usted intentó el coito, durante el mes pasado, ¿con qué frecuencia se implicó en otras prácticas sexuales (besos, caricias y roces)?

En cuanto a la frecuencia de implicación en otra práctica sexual, además del coito, como caricias, besos o roces durante el mes pasado, de los 12 hombres, 5 niegan haberse implicado en ninguna, 4 afirman haberse implicado una o más de una vez al día, uno las ha llevado a cabo una vez durante el mes pasado, otro hombre afirma que se ha implicado en este tipo de práctica sexuales dos o tres veces el mes pasado y aparece un caso de implicación en dichas prácticas una vez por semana.

En cuanto a las mujeres, 4 responden que "nunca" se han implicado en este tipo de prácticas sexuales durante el último mes, otras 4 mujeres afirman haber mantenido estas prácticas sexuales varias veces al mes, una mujer lo ha hecho más de una vez al día y otra afirma mantener estas prácticas sexuales una vez al mes.

Comparado con hace un año, ¿ha cambiado la frecuencia general de sus prácticas sexuales?

Las respuestas a este ítem se codifican en una escala de 1 al 5, siendo 1 "Ha aumentado mucho" y 5 "На disminuido mucho". La media de respuesta de la muestra masculina es de $4 \quad(\mathrm{DT}=0.943)$, siendo la mínima 3, lo que indica que ninguno de los hombres ha afirmado que la frecuencia de sus prácticas hayan aumentado. En general, la frecuencia de las prácticas sexuales de las mujeres "ha disminuido moderadamente" (60\%) o "ha disminuido mucho" $(30 \%)$, existiendo un único caso que afirma que no haber notado cambios en la frecuencia de sus prácticas sexuales. Cerca del $42 \%$ de los hombres afirma que la 
frecuencia de sus prácticas sexuales "ha disminuido mucho", aquellos que afirman que "ni han aumentado ni disminuido" son el $41 \%$ y el $16,6 \%$ dice que esta frecuencia ha disminuido, pero "moderadamente".

\section{Diferencias en la actividad sexual en función del lugar de residencia.}

En respuesta al tercer objetivo, realizamos una comparación entre las respuestas dadas al ítem En el último año, ¿ha tenido usted alguna práctica sexual (sexo coital, masturbación, roces o caricias)? por las personas residentes en una institución y las que residen en su propio domicilio. Para ello llevamos a cabo una prueba no paramétrica de comparación de proporciones para dos o más muestras independientes, dado que los datos se ajustan a la distribución de chi cuadrado, la variable dependiente es nominal y nos permite comparar dos o más distribuciones de proporciones, determinando si la diferencia no se debe al azar, es decir, que sea estadísticamente significativa.

Con valor de $\mathrm{p}=.008$ para el estadístico Fisher se encuentran diferencias significativas en la actividad sexual del último año entre las mujeres que viven en una residencia $(\mathrm{n}=1,10 \%)$ y las que viven en su domicilio familiar $(n=9,90 \%)$ (Véase Figura 1$)$.

\section{En el último año, ¿ha tenido alguna práctica sexual?}

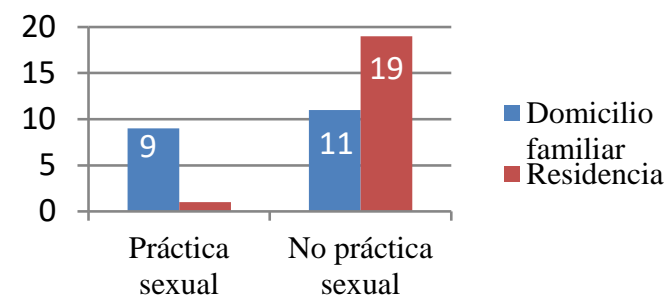

Figura 1. Práctica sexual en el último año entre las mujeres que viven en residencia o en su domicilio.

En cuanto a la muestra masculina, podemos observar la proporción de hombres que han mantenido relaciones sexuales en el último año entre los que viven en una residencia $(n=4,33.3 \%)$ y los que viven en su domicilio familiar $(n=8,66.7 \%)$.

Con valor de $p=.081$ para el estadístico Fisher podemos comprobar que no existen diferencias entre las respuestas dadas por los hombres que viven en residencia y los que viven en su domicilio (Véase Figura 2).

\section{En el último año, ¿ha tenido alguna práctica sexual?}

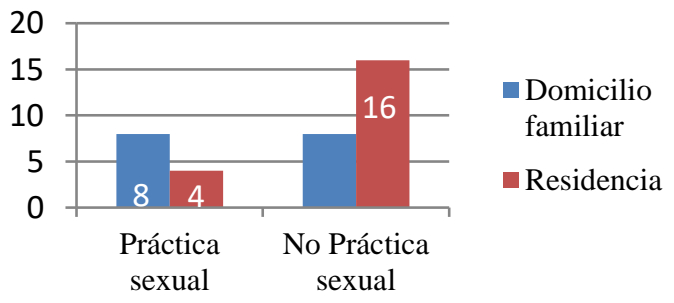

Figura 2. Práctica sexual en el último año entre los hombres que viven en residencia o en su domicilio.
Nivel de satisfacción de las personas mayores con la vida sexual.

En respuesta al cuarto objetivo realizamos un análisis descriptivo de las respuestas al enunciado que pregunta sobre el nivel de satisfacción de su vida sexual en los últimos tres meses, siendo la escala de 1 "muy satisfecho" a 5 "muy insatisfecho"

El análisis de las respuestas dadas por los hombres al enunciado Durante los últimos tres meses, ¿cómo de satisfecho ha estado usted con su vida sexual general?, 6 de ellos afirman estar "muy satisfechos" con su vida sexual general y sólo uno contesta "moderadamente satisfecho". Cuando analizamos las respuestas de la muestra femenina al ítem sobre la satisfacción con la frecuencia de su práctica sexual, de las mujeres 7 que contestaron al mismo, 4 contestan que están "muy satisfechas", 2 "moderadamente satisfechas", y una de ellas afirma que "ni satisfecha ni insatisfecha"

\section{Discusión}

Los resultados de nuestro estudio indican que la mayoría de los hombres de nuestra muestra tienen una actitud hacia la sexualidad entre las personas mayores que no se corresponde con la visión viejista que se tiene sobre el pensamiento de los mismos por parte del resto de la sociedad. Por el contrario, en la muestra femenina aparece un porcentaje más alto de aquellas con una visión de la sexualidad entre las personas mayores más estigmatizada, tal y como aparece también en otros estudios (García, 2000; Leyva-Moral, 2008).

Muchos de los estereotipos que se mantienen en nuestra sociedad actual, aparecen en las actitudes de las personas mayores que conforman nuestra muestra. Por ejemplo, tener relaciones sexuales antes del matrimonio o tenerlas con otra persona que no sea su pareja, se considera como algo malo. Incluso a la hora de juzgar la capacidad de disfrutar del sexo, aparece un pensamiento estereotipado como pensar que los hombres tienen de forma natural un mayor deseo sexual que las mujeres. La falsa creencia de que las mujeres mayores interesadas en el sexo son las que en su juventud eran adictas a él (Leyva-Moral, 2008) puede hacer creer a las mujeres que lo que se dice es cierto y condicionar su comportamiento sexual.

En cuanto a la relación entre la capacidad de tener relaciones y la edad, tanto los hombres como las mujeres consideran que a medida que vamos envejeciendo la frecuencia de las mismas disminuye. Los estereotipos que relacionan la sexualidad con la vejez aparecen cuando se considera esta etapa para las personas mayores como un proceso irreversible que produce un deterioro físico y psicológico debido a los cambios fisiológicos progresivos que deterioran la capacidad sexual, lo que induce a la población a creer que entre los mayores hay falta de deseo sexual, tratando a la «tercera edad» como sinónimo de asexuación (García, 2005). Sin embargo, en la mayoría de las personas que conforman las dos muestras hay una coincidencia a la hora de considerar que las relaciones sexuales satisfactorias se relacionan con el mantenimiento de una relación a largo plazo, siendo 
esta actividad sexual esencial para la pareja. En cuanto a esto, gran parte de la muestra afirma que los cambios sexuales son importantes para la gente mayor, así como que la actividad sexual es un aspecto beneficioso física y psicológicamente, negando la falsa creencia, tal y como afirma Leyva-Moral (2008) de que la actividad sexual durante la vejez puede ser perjudicial para la salud.

Como ya hemos mencionado anteriormente, una de las creencias más vinculadas con las personas mayores es el hecho de que el sexo ha dejado de tener interés para las mismas. En el segundo objetivo del presente trabajo de investigación nos planteábamos conocer la frecuencia de las prácticas sexuales del último año. Los resultados indican que en el caso de los hombres son 12 (lo que supone el 33.3\% de la muestra) los que afirman haber mantenido prácticas sexuales y en el caso de las mujeres, son 10 (25\% de la muestra) las que lo afirman. Estos datos nos indican que no son ciertas las afirmaciones de que en la vejez no se mantienen prácticas sexuales. Si bien debemos confirmar que la frecuencia de las prácticas sexuales disminuye con la edad.

Con nuestro tercer objetivo pretendíamos conocer si el lugar de residencia de la persona mayor podría condicionar o no su actividad sexual. En un trabajo de investigación realizado por Spetor y Femeth (1996) encontraron que tan solo el $10 \%$ de las personas que vivían en una residencia mantenían actividades sexuales. Nuestros resultados siguen la misma dirección, son pocos los hombres y mujeres que viviendo en una residencia continúan con estas prácticas. Los resultados de los análisis con respecto a este objetivo nos indican que no existen diferencias entre los hombres que viven en residencia y los que viven en su domicilio familiar, pero sí las hay entre la actividad sexual de las mujeres que viven en residencia y las que viven en su domicilio familiar. De esta manera, la hipótesis planteada sobre el hecho de que vivir en una residencia condicionaría las relaciones sexuales es cierta, pero solo para la muestra femenina.

Por último, partiendo de la importancia que para este grupo etario tienen los aspectos afectivos, hemos querido conocer su nivel de satisfacción con su vida sexual general, independientemente de si han aumentado o no. Como afirma Murgieri (2011), para este colectivo son importantes las relaciones de calidad, comunicativas y armónicas, al margen de la cantidad. Nuestros resultados indican que la mayoría de las personas que han contestado a este ítem (hombre y mujeres) afirma estar muy satisfechos o moderadamente satisfechos con su vida sexual general. Debemos, pues, rechazar la hipótesis planteada en cuanto a la insatisfacción con su vida sexual general.

\section{Referencias bibliográficas}

Fernández-Ballesteros, Oris y Walker (2006). Una vejez activa en España. Recuperado de http://digital.csic.es/bitstream/10261/69327/1/2012Ve jezActiva.pdf
Freire, C. y Ferradás, M. (2016). Calidad de vida y bienestar en la vejez. Madrid: Editorial Pirámide.

García, J. L. (2000). Educación sexual y afectiva en personas con minusvalías psíquicas. Cádiz: Asociación Down Lejeune.

García, J. L. (2005). La sexualidad y la afectividad y en la vejez. Informes Portal Mayores, 41. Madrid: Portal Mayores. Recuperado de http://www.imsersomayores.csic.es/documentos/docu mentos/garcia-sexualidad-01.pdf

Lee, D., Nazroo, J., O’Connor, D., Blake, M. y Pendleton, N. (2016). Sexual Health and Well-being Among Older Men and Women in England: Findings from the English Longitudinal Study of Ageing. Archives of Sexual Behavior, 45(1), 133-144. doi:10.1007/s10508-014-0465-1

Leyva-Moral, J. M. (2008). La expresión sexual de los ancianos. Una de falsos mitos. Index de Enfermería, 17 (2), 124-127.

Mitchell, K. R., Ploubidis, G. B., Datta, J., y Wellings, K. (2012). The Natsal-SF: A validated measure of sexual function for use in community surveys. European Journal of Epidemiology, 27(6), 409-418.

Murgieri, J. (2011). Erótica, sexualidad y vejez en una institución geriátrica. Revista electrónica puc-sp, 14(5), 151-161.

O’Connor, D. B., Corona, G., Forti, G., Tajar, A., Lee, D. M., Finn, J. D. y Wu, F. C. (2008). Assessment of sexual health in aging men in Europe: Development and validation of the European Male Ageing Study sexual function questionnaire. Journal of Sexual Medicine, 5(6), 1374-1385.

Pérez, L. (2006). La estructura social de la vejez en España. Nuevas y viejas formas de envejecer. Ministerio de Trabajo y Asuntos Sociales. Secretaría de Estado de Servicios Sociales, Familias y Discapacidad. Instituto de Mayores y Servicios Sociales. Madrid: IMSERSO.

Spector, I. P. y Femeth, S. M. (1996). Sexual behaviors and attitudes of geriatric residents in long-term care facilities. Journal Sex \& Marital Therapy, 22, 235246.

Waite, L. J., Laumann, E. O., Das, A., y Schumm, L. P. (2009). Sexuality: Measures of partnerships, practices, attitudes, and problems in the National Social Life, Health, and Aging Study. Journals of Gerontology Series B: Psychological Sciences and Social Sciences, 64(1), 56-66. 